\title{
Curvature-induced spin-orbit coupling and spin relaxation in a chemically clean single-layer graphene
}

\author{
Jae-Seung Jeong* \\ School of Physics, Korea Institute for Advanced Study, Seoul 130-722, Korea \\ Jeongkyu Shin and Hyun-Woo Lee \\ Department of Physics and PCTP, Pohang University of Science and Technology, Pohang 790-784, Korea
}

(Dated: October 31, 2018)

\begin{abstract}
The study of spin-related phenomena in materials requires knowledge of the precise form of effective spin-orbit coupling for conducting carriers in solid-states systems. We demonstrate theoretically that curvature induced by corrugations or periodic ripples in single-layer graphenes generates two types of effective spin-orbit couplings. In addition to the spin-orbit coupling reported previously that couples with sublattice pseudospin and corresponds to the Rashba-type spin-orbit coupling in a corrugated single-layer graphene, there is an additional spin-orbit coupling that does not couple with the pseudospin, which can not be obtained from the extension of the curvature-induced spin-orbit coupling of carbon nanotubes. Via numerical calculation we show that both types of the curvature-induced spin-orbit coupling make the same order of contribution to spin relaxation in chemically clean single-layer graphene with nanoscale corrugation. The spin relaxation dependence on the corrugation roughness is also studied.
\end{abstract}

\section{INTRODUCTION}

Graphene has attracted much interest due to its unusual linear energy spectrum and electronic properties.1 It is also a promising material for spin-based applications since spin relaxation (SR) in graphenes is expected to be weak due to the suppression of the hyperfine interaction of ${ }^{12} \mathrm{C}$ and the weak atomic spin-orbit coupling (SOC). Various spintronic devices ${ }^{2}$ and spin qubits ${ }^{3}$ on graphenes have been suggested.

Recent experiments, however, measured SR times (SRTs) of the order of $0.1-0.5 \mathrm{~ns}, \frac{4}{4}$ which were much shorter than expected and comparable to other materials such as aluminum ${ }^{5}$ and copper ${ }^{6}$ Those experiments stirred up intensive theoretical investigation of the SR in single-layer graphene (SG) to clarify the origin of the short SRT. SG samples in Ref. ${ }^{[}$have low mobility $\sim 2000 \mathrm{~cm}^{2} / \mathrm{Vs}$ and the SRT was found to increase with increasing carrier density, which is consistent with the Elliot-Yafet (EY) SR mechanism ${ }^{7}$ arising from the interplay of SOC and momentum scattering. However, it is not clear what kind of SOC is mainly responsible for the $\mathrm{SR}$ in SG, and various kinds of SOCs are examined. It was suggested ${ }^{8}$ that adatoms on SG can enhance the local SOC to the order of $10 \mathrm{meV}$, which results in short SRT comparable to the experimental value 4 for low mobility SG samples. Thus the adatom-induced SOC provides a possible origin ${ }^{911}$ of the short SRT in Ref. ${ }^{4}$. If this SOC is indeed the main origin, which requires further confirmation, it implies that the SRT may be enhanced in SGs without adatoms. To find out how greatly the SRT can be enhanced, various factors limiting the SRT should be examined carefully. In exfoliated SGs, charged impurities or surface phonons in the substrate ${ }^{9}$ can induce the effective SOC of the order of $0.01 \mathrm{meV}$. Also, effective SOC of the order of $0.01-0.1 \mathrm{meV}^{\sqrt{10}}$ can be induced by local curvature effects arising from corrugations 12 and periodic ripples $18 / 19$ observed in suspended $12 \mid 17 / 18$ and exfoliated 13 SGs. Each substrate-induced and curvature-induced SOC combined with momentum scattering off impurities 1120 or electron-electron Coulomb scattering, 21 and substrate-induced or curvature-induced SOC itsell ${ }^{22}$ can cause SR. The estimated SRTs are at least of the order of $10 \mathrm{~ns}$, which is about two order of magnitude larger than measured SRTs.

For a more reliable investigation of spin-related phenomena such as SR and spin coherence, it is important to know the precise form of SOC. In this respect, existing studies of the curvature-induced SOC in SGs are not satisfactory, since the precise form of this SOC is not known. Previous theoretical studies $10 \mid 20-22$ assumed a particular form of the SOC inferred from that of a carbon nanotube (CNT) ${ }^{10}$ However the SOC-induced energy splitting measurement ${ }^{23}$ in ultra-clean CNTs revealed that

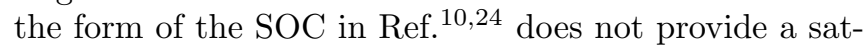
isfactory description of the SOC effect in a CNT. Later theoretical works ${ }^{25 \mid 26}$ reported that the correct form of the SOC in a CNT has an additional term in addition to the SOC form in Ref! $10 \mid 24$. The additional term does not couple to the pseudospin degrees of freedom in a CNT and thus differs qualitatively from the previously known SOC term, $\frac{1024}{10}$ which couples to the pseudospin. It was demonstrated in Ref. ${ }^{26}$ that the interplay of the two SOC terms can explain the SOC-induced energy splitting measurement. ${ }^{23127}$ It is then reasonable to expect that a similar additional SOC term may exist for SGs as well.

Finding the correct curvature-induced SOC can have an implication not only on SR in the current experiments, 4 but also on SR in chemically clean SGs. As technology for the preparation of SGs progresses so as to diminish impurity effects, the fundamental limit of SR would be governed by intrinsic sources. Recalling that atomically flat SGs are thermodynamically unstable, 28 the curvature-induced SOC is expected to be one of the 
intrinsic origins determining the upperbound of the SRT in chemically clean SGs. 29 We believe that this expectation is very plausible since SGs and CNTs are very similar chemically and the dominant source of the SOC in ultra-clean CNTs $s^{23 \mid 27}$ is the curvature-induced SOC.

In this paper, we show that in addition to the curvature-induced Rashba-type SOC reported in an existing theory, 10 which couples to the pseudospin, there exists an additional type of the curvature-induced SOC, which does not couple to the pseudospin and thus appears as the diagonal term in the pseudospin representation. In this respect, this additional SOC term is similar to the additional SOC term in CNTs. ${ }^{25 \mid 26}$ However these two additional terms have topologically different origins since the periodicity in the circumferential direction arising from the tube topology of CNTs is absent in SGs. Thus the additional SOC term in SGs cannot be obtained from the additional SOC term in CNTs through a trivial extension. This point was not addressed in an earlier attempt $\mathrm{t}^{26]}$ to derive the curvature-induced SOC in SGs. In this paper, we also examine the SR due to the curvature in chemically clean SGs and show that the effect of the additional SOC on the SR is comparable in magnitude to the effect of the previously known curvature-induced SOC. Thus the additional curvature-induced SOC term should be included in studies of spin-related phenomena in SGs. We also investigate SR dependence on the fractal dimension of the corrugation roughness, 14 and show that corrugation roughness affects the SR.

This paper is organized as follows. In Sec. II] we show analytical expressions of two types of the curvatureinduced SOCs in SGs, and then demonstrate how to obtain them microscopically using tight-binding (TB) Hamiltonian of the local curvature effect and the atomic SOC of carbon. In Sec. III, we investigate effects of the effective SOC on SR based on the kinetic equation of the spin density operator in corrugated SGs, and calculate numerically SRT. We give a brief summary in Sec. IV

\section{EFFECTIVE SPIN-ORBIT COUPLING}

In this section, we present the effective Dirac Hamiltonian in flat SGs, and then, the effective SOC Hamiltonian arising from the local curvature effect combined with the atomic SOC in corrugated SGs. After that, we demonstrate how to evaluate the effective SOC using the TB Hamiltonian via second-order perturbation theory, and why the SOC can not be inferred from the extension of that of CNTs.

\section{A. Analytic expression}

Graphene honeycomb lattice with two sublattices $A$ and $B$ (Fig. 1), has $\pi$-band consisting of $p_{z}$ orbitals near the Fermi level situated at hexagonal corners in the Brillouin zone. Close to the Dirac point $\mathbf{K}_{1}$ and
$\mathbf{K}_{2}=-\mathbf{K}_{1}, \pi$-band states with wave vector $\mathbf{k}=\left(k_{x}, k_{y}\right)$ $\left[|\mathbf{k}| \ll\left|\mathbf{K}_{1(2)}\right|\right]$ relative to the $\mathbf{K}_{1(2)}$, can be described by the effective Dirac Hamiltonian $\mathcal{H}_{\text {Dirac }}$. When the two sublattices $A$ and $B$ of the honeycomb lattices are used as bases, the $\mathcal{H}_{\text {Dirac }}$ is written as 31

$$
\mathcal{H}_{\text {Dirac }}=\hbar v_{F}\left(\begin{array}{cc}
0 & e^{i \tau_{z} \alpha}\left(\tau_{z} k_{x}-i k_{y}\right) \\
e^{-i \tau_{z} \alpha}\left(\tau_{z} k_{x}+i k_{y}\right) & 0
\end{array}\right)
$$

where $v_{F}$ is the Fermi velocity, and $\tau_{z}= \pm 1$ denotes the $\mathbf{K}_{1(2)}$. Here, the angle $\alpha$ is defined counterclockwise from the $\hat{\mathbf{y}}$ direction and the $\mathrm{C}-\mathrm{C}$ bond vector $\mathbf{b}_{\mathrm{cc}}$-direction (Fig. 1).

Local curvature effects arising from smooth corrugations or ripples in SGs combined with atomic SOC of carbon $^{32}$ generate the effective SOC for the $\pi$-band states 10 The corrugated SG can be described as a surface $z=h(\mathbf{r})$ with height $h(\mathbf{r})$ as a function of spatial position $\mathbf{r}=(x, y)$ in the two-dimensional (2D) reference plane (Fig. 2). Experiments 12 15 17 suggest that $h(\mathbf{r})$ shows fluctuations of the order of $1 \mathrm{~nm}$ over scales $\sim 10 \mathrm{~nm}$, which can be specified by $h_{i} h_{j} \ll 1(i, j=x, y)$ with $h_{i}$ being the partial derivative along the $i$ direction. When the local structure of the SG surface has two principal curvatures $\kappa_{1}(\mathbf{r})$ and $\kappa_{2}(\mathbf{r})$, we find that the $\mathcal{H}_{\text {soc }}(\mathbf{r})$ is written as

$$
\mathcal{H}_{\mathrm{soc}}(\mathbf{r})=\left(\begin{array}{cc}
\zeta^{\prime} \tau_{z} \boldsymbol{\mu}(\mathbf{r}) \cdot \mathbf{s} & \zeta e^{i \tau_{z} \alpha} \nu(\mathbf{r})\left(\tau_{z} s_{y}+i s_{x}\right) \\
\zeta e^{-i \tau_{z} \alpha} \nu(\mathbf{r})\left(\tau_{z} s_{y}-i s_{x}\right) & \zeta^{\prime} \tau_{z} \boldsymbol{\mu}(\mathbf{r}) \cdot \mathbf{s}
\end{array}\right)
$$

where $\mathbf{s}=\left(s_{x}, s_{y}, s_{z}\right)$ denotes the real spin Pauli matrix. Here, $\zeta$ and $\zeta^{\prime}$ are material parameters, and $\boldsymbol{\mu}(\mathbf{r})$ and $\nu(\mathbf{r})$ are geometrical parameters containing the local curvature information. For $\alpha=0$, the off-diagonal part of Eq. (2) reduces to the Rashba-type SOC $\propto$ $\left(\sigma_{y} s_{x}-\tau_{z} \sigma_{x} s_{y}\right)$ reported in existing theories ${ }^{10 \mid 20}$, where $\boldsymbol{\sigma}=\left(\sigma_{x}, \sigma_{y}, \sigma_{z}\right)$ denotes the sublattice-pseudospin Pauli matrix. On the other hand, the diagonal part of Eq. (2) is one of main results of this paper, which was not ignored

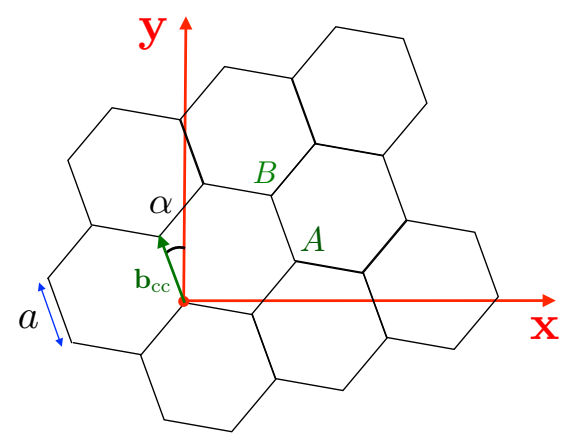

FIG. 1. (Color online) Single-layer flat graphene honeycomb lattice with two sublattices $A$ and $B$. The angle $\alpha$ is defined counterclockwise from the $\hat{\mathbf{y}}$ to the $\mathrm{C}-\mathrm{C}$ bond vector $\mathbf{b}_{\mathrm{cc}} \cdot a=$ $\left|\mathbf{b}_{\mathrm{cc}}\right| \approx 1.42 \AA$ is the carbon-carbon distance. 
in previous studies. Note that this additional SOC term in the diagonal part does not couple to the pseudospin.

The material parameters $\zeta$ and $\zeta^{\prime}$ are, respectively, given by $\zeta=a \lambda_{\mathrm{so}}\left(\epsilon_{p}-\epsilon_{s}\right)\left(V_{p p}^{\pi}+V_{p p}^{\sigma}\right) /\left(12 V_{s p}^{\sigma 2}\right)$ and $\zeta^{\prime}=$ $a \lambda_{\mathrm{so}} V_{p p}^{\pi} /\left(2\left(V_{p p}^{\pi}-V_{p p}^{\sigma}\right)\right)$, where $\lambda_{\text {so }}$ is the atomic SOC strength of the $p$ orbitals, $\epsilon_{s(p)}$ is the atomic energy for $s(p)$ orbitals and $a$ is the carbon-carbon distance. Here, $V_{s p}^{\sigma}$ and $V_{p p}^{\pi(\sigma)}$ represent the coupling strength in the absence of the curvature for the $\sigma$ coupling between nearestneighbor $s$ and $p$ orbitals and the $\pi(\sigma)$ coupling between nearest-neighbor $p$ orbitals, respectively.

The geometrical parameters $\nu(\mathbf{r})$ and $\boldsymbol{\mu}(\mathbf{r})$ are given as follows. $\nu(\mathbf{r})$ is a sum of the two local curvatures, $\nu(\mathbf{r})=\kappa_{1}(\mathbf{r})+\kappa_{2}(\mathbf{r})$ and $\boldsymbol{\mu}(\mathbf{r})$ is a weighted sum of the two local curvatures,

$$
\begin{aligned}
\boldsymbol{\mu}(\mathbf{r}) & =\left(\mu_{x}(\mathbf{r}), \mu_{y}(\mathbf{r})\right) \\
& =\sum_{j=1}^{2} \kappa_{j}(\mathbf{r})\left(\sin \left[2 \theta_{j}(\mathbf{r})-3 \alpha\right], \cos \left[2 \theta_{j}(\mathbf{r})-3 \alpha\right]\right),
\end{aligned}
$$

where $\theta_{1}(\mathbf{r})$ and $\theta_{2}(\mathbf{r})$ are the angles between a local $\mathbf{x}^{\prime}(\mathbf{r})$-direction and the two principal curvature directions (see Fig. 2 for their precise definitions). Since the two principal curvature directions are mutually orthogonal, $\theta_{2}(\mathbf{r})=\theta_{1}(\mathbf{r}) \pm \pi / 2$.

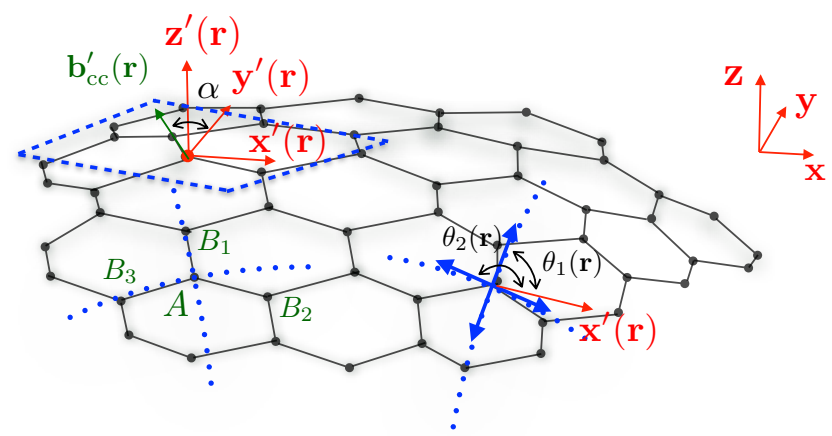

FIG. 2. (Color online) Schematic of a partial convex structure in a corrugated SG surface described by $z=h(\mathbf{r})$ where $\mathbf{r}=(x, y)$ within the $2 \mathrm{D}$ reference $x y$-plane. The $\hat{\mathbf{z}}^{\prime}(\mathbf{r})$ is the unit vector normal to the local tangential plane and the $\mathbf{b}_{\mathrm{cc}}^{\prime}(\mathbf{r})$ (green arrow) is the projected vector from the local $\mathrm{C}-\mathrm{C}$ bond onto the tangent plane specified by the (blue) dashed quadrangle normal to the $\hat{\mathbf{z}}^{\prime}(\mathbf{r})$. The $\mathbf{y}^{\prime}(\mathbf{r})$-direction in the tangent plane is defined to make the angle $\alpha$ [Fig. 1] clockwise from the $\mathbf{b}_{\mathrm{cc}}^{\prime}(\mathbf{r})$ in the tangent plane. Then the $\mathbf{x}^{\prime}(\mathbf{r})$-direction is determined automatically to be mutually orthogonal to $\mathbf{z}^{\prime}(\mathbf{r})$ direction and $\mathbf{y}^{\prime}(\mathbf{r})$-direction. Dotted (blue) lines are curvature lines tangent to principal directions along orthogonal (blue) arrows where the direction of arrowheads is arbitrary. The angle $\theta_{1(2)}(\mathbf{r})$ is defined by $\mathbf{x}^{\prime}(\mathbf{r})$-direction and the principal directions, and $\theta_{2}(\mathbf{r})=\theta_{1}(\mathbf{r}) \pm \pi / 2$. The sublattice $A$ and its nearest-neighbor sites $B_{l}(l=1,2,3)$ with two curvature lines are indicated.
Both diagonal and off-diagonal terms in Eq. 22 are invariant under the time reversal symmetry. The offdiagonal term is allowed when the mirror symmetry with respect to the $x y$-plane is broken ${ }^{33}$ On top of that, owing to the broken $C_{3}$ symmetry in corrugated SGs, the diagonal term of the form $\tau_{z} s_{x(y)}$ is allowed as well. Note that the diagonal term has the $\theta_{1(2)}(\mathbf{r})$-dependence. However, it can not be obtained from the naive extension of the diagonal SOC of CNTs that depends on the chiral angle $\theta$ through $\cos (3 \theta) \stackrel{25 \mid 26}{.}$ Quantitatively, both terms have the same order of magnitude. Using $\lambda_{\text {so }} \approx 10 \mathrm{meV}, \underline{32}$ $\epsilon_{s}=-7.3 \mathrm{eV}, \epsilon_{p}=0 \mathrm{eV}, V_{s p}^{\sigma}=4.20 \mathrm{eV}, V_{p p}^{\sigma}=5.38 \mathrm{eV}$, $V_{p p}^{\pi}=-2.24 \mathrm{eV}$ in Ref. ${ }^{34}$ we obtain $\zeta^{\prime} \approx 0.21 \mathrm{meVnm}$ and $\zeta \approx 0.15 \mathrm{meVnm}$.

The $\theta_{j}(\mathbf{r})$ and the $\alpha$-dependence of $\mathcal{H}_{\text {soc }}(\mathbf{r})$ combined with the SG morphology have interesting implications. First of all, since $\theta_{2}(\mathbf{r})=\theta_{1}(\mathbf{r}) \pm \pi / 2$, the diagonal term depends on the difference between two principal curvatures in contrast to the off-diagonal term depending on their sum. As a result, at umbilical points where $\kappa_{1}(\mathbf{r})=\kappa_{2}(\mathbf{r})$, the diagonal term disappears while the off-diagonal term remains, which confirms that every tangent vector can be a principal direction at the umbilical points. ${ }^{35}$ In contrast with the umbilical points, at saddle points where $\kappa_{1}(\mathbf{r})=-\kappa_{2}(\mathbf{r})$, the diagonal term only appears while the off-diagonal term disappears.

In addition, for periodic ripples having unidirectional curvature direction $\left(\kappa_{1}(\mathbf{r}) \neq 0\right.$ and $\left.\kappa_{2}(\mathbf{r})=0\right)$, the diagonal SOC field direction varies depending on the principal curvature direction and the honeycomb lattice orientation as well. When $\alpha=q / 3(q \in \mathbb{Z})$ so that the $\hat{\mathbf{x}}$ is along the zigzag direction [Fig. 1], for example, the diagonal term is $\propto \kappa_{1}(\mathbf{r}) s_{y}$ for $\theta_{1}(\mathbf{r})=0$ and $\pi / 2$, while it is $\propto \kappa_{1}(\mathbf{r}) s_{x}$ for $\theta_{1}(\mathbf{r})=\pi / 4$. On the other hand, when $\alpha=q / 3+\pi / 6$ so that the $\hat{\mathbf{x}}$ is along the armchair direction [Fig.1], the diagonal term is $\propto \kappa_{1}(\mathbf{r}) s_{x}$ for $\theta_{1}(\mathbf{r})=0$ and $\pi / 2$, while it is $\propto \kappa_{1}(\mathbf{r}) s_{y}$ for $\theta_{1}(\mathbf{r})=\pi / 4$. Recent experiments shows that the periodic ripple line direction can be controlled ${ }^{18}$ and the multiple periodic ripple domains with different ripple lines can occur, 19 which implies that the variation of the diagonal term depending on the curvature direction and the honeycomb lattice orientation could be significant for the analysis of spin transport in SGs with those structural deformation. After all, in order to describe precisely the curvatureinduced SOC in SGs both terms should be considered on an equal footing.

The $\mathcal{H}_{\text {soc }}(\mathbf{r})$ can be written as an explicit function of $h(\mathbf{r})$ in the SG surface, $z=h(\mathbf{r})$ where $h_{i} h_{j} \ll 1$ $(i, j=x, y)$. The principal curvature is the eigenvalue of the shape operator $\mathcal{S}=\mathcal{F}_{1}^{-1} \mathcal{F}_{2}$ with $\mathcal{F}_{1(2)}$ being the first (second) fundamental form ${ }^{35}$ Moreover, since its eigenvector $\hat{\boldsymbol{v}}_{1(2)}(\mathbf{r})$ is along the principal direction, $\theta_{1(2)}(\mathbf{r})$ can be determined by the $\hat{\boldsymbol{v}}_{1(2)}(\mathbf{r})$ and $\hat{\mathbf{x}}^{\prime}(\mathbf{r})$, which is assumed as $\hat{\mathbf{x}}^{\prime}(\mathbf{r}) \approx\left(1,0, h_{x}\right)$ in the zeroth order of $h_{i} h_{j}$. $\mathcal{F}_{1}$ and $\mathcal{F}_{2}$ are, respectively, given by ${ }^{35}$ 
$\mathcal{F}_{1}=\left(\begin{array}{cc}1+h_{x}^{2} & h_{x} h_{y} \\ h_{x} h_{y} & 1+h_{y}^{2}\end{array}\right), \mathcal{F}_{2}=\frac{1}{\sqrt{1+|\nabla h|^{2}}}\left(\begin{array}{cc}h_{x x} & h_{x y} \\ h_{x y} & h_{y y}\end{array}\right)$

and then, the $\kappa_{1(2)}(\mathbf{r})$ and $\hat{\boldsymbol{v}}_{1(2)}(\mathbf{r})$ are written as $\kappa_{1(2)}(\mathbf{r}) \approx\left(h_{x x}+h_{y y}-(-1)^{1(2)}\left[\left(h_{x x}-h_{y y}\right)^{2}+4 h_{x y}^{2}\right]^{\frac{1}{2}}\right) / 2$ and $\hat{\boldsymbol{v}}_{1(2)}(\mathbf{r}) \approx\left[\alpha_{1(2)} \hat{\mathbf{x}}+\beta \hat{\mathbf{y}}+\left(\alpha_{1(2)} h_{x}+\beta h_{y}\right) \hat{\mathbf{z}}\right] / \mathcal{N}$, respectively, where $\alpha_{1(2)} \approx h_{x x}-h_{y y}-(-1)^{1(2)}\left[\left(h_{x x}-h_{y y}\right)^{2}+\right.$ $\left.4 h_{x y}^{2}\right]^{\frac{1}{2}}$ and $\beta \approx 2 h_{x y}$ with normalization constant $\mathcal{N}$.

\section{B. Tight-binding Hamiltonian}

Now we demonstrate how to derive Eq. 22 microscopically. Using second-order perturbation theory ${ }^{36}$ with the local curvature and the atomic SOC as weak perturbation, we obtain Eq. (2). Thus we need to know the TB Hamiltonian of the local curvature $H_{\mathrm{c}}$ and the atomic SOC $H_{\text {so }}$ taking the honeycomb lattice structure into account exactly. Most parts of this subsection are devoted to the TB theory of the $\pi-\sigma$ hybridization among $s$ and $p$ orbitals. The unoccupied $d$ orbital effects $\frac{3738}{}$ are discussed at the end of this subsection.

Firstly, $H_{\mathrm{c}}$ is determined purely by local curvature effect dependent on $\kappa_{1(2)}(\mathbf{r})$ and $\theta_{1(2)}(\mathbf{r})$ at three bonds between an atomic site $\mathbf{r}_{A}$ in the sublattice $A$ and nearestneighbor sites $B_{l}(l=1,2,3)$ in the sublattice $B$ [Fig. 2]. For the smoothly corrugated SGs, we consider solely one of two principal curvature effects assuming that the other principal curvature effect is absent. There are also mutual curvature effects between them, but those effects can be ignored for the smooth corrugated surface with

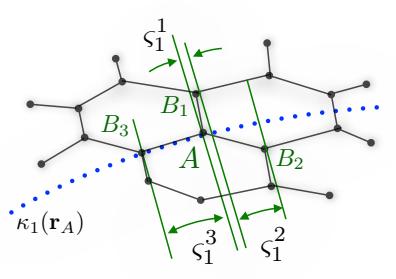

(a)

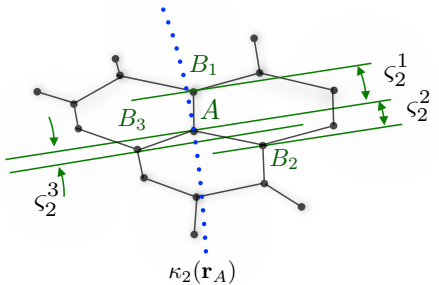

(b)
FIG. 3. (Color online) Schematic of a partial convex SG surface with sublattices $A$ and $B_{l}(l=1,2,3)$ indicated in Fig. 2 which is assumed to have unidirectional principal curvature. Although the original surface [Fig. 2] has finite principal curvatures $\kappa_{1}\left(\mathbf{r}_{A}\right)$ and $\kappa_{2}\left(\mathbf{r}_{A}\right)$, each curvature effect is evaluated under the assumption that the other principal curvature is zero. $\kappa_{1}\left(\mathbf{r}_{A}\right) \neq 0$ and $\kappa_{2}\left(\mathbf{r}_{A}\right)=0$ in (a) and $\kappa_{1}\left(\mathbf{r}_{A}\right)=0$ and $\kappa_{2}\left(\mathbf{r}_{A}\right) \neq 0$ in (b). Dotted (blue) lines represent curvature lines, and principal direction at $\mathbf{r}_{A}$ is tangent to the curvature lines. The arc length $\varsigma_{j}^{l}(j=1,2)$ between a solid (green) line passing $A$ atom normal to the principal direction, and its parallel solid (green) line passing $B_{l}$ atom, is related with $\omega_{j}^{l}$ by $\omega_{j}^{l} \approx \varsigma_{j}^{l} \kappa_{j}(\mathbf{r}) / 2$ [Eq. (5)], $h_{i} h_{j} \ll 1(i, j=x, y)$ since they are higher order in $a / \mathcal{R}_{1(2)}\left(\mathbf{r}_{A}\right)$ where $\mathcal{R}_{1(2)}\left(\mathbf{r}_{A}\right)=\kappa_{1(2)}^{-1}\left(\mathbf{r}_{A}\right)$. Then, it is enough to deal with two unidirectional ripple structures whose principal directions are orthogonal as shown in Fig. 3. For the unidirectional ripple structure, each principal curvature and the angle between the principal direction and the $\mathbf{x}^{\prime}(\mathbf{r})$ at three neighbor $\mathrm{C}-\mathrm{C}$ bonds around the $\mathbf{r}_{A}$ are same as $\kappa_{1(2)}\left(\mathbf{r}_{A}\right)$ and $\theta_{1(2)}\left(\mathbf{r}_{\mathbf{A}}\right)$, respectively. Then, up to first order in $a / \mathcal{R}_{1(2)}\left(\mathbf{r}_{A}\right)$, the $H_{\mathrm{c}}$ can be approximated as $H_{\mathrm{c}} \approx H_{1}+H_{2}$, each of which describes one principal curvature effect in the absence of the other one. Two principal curvatures yield correlation effects between term in $H_{\mathrm{c}}$, but they are higher order in term of $a / \mathcal{R}_{1(2)}(\mathbf{r})$ and thus can be ignored. The $H_{j}(j=1,2)$ is written as 10126

$$
\begin{aligned}
H_{j} & =\sum_{\mathbf{r}_{A}} \sum_{l=1}^{3} \sum_{\eta=\uparrow, \downarrow}\left[-S_{j}^{l}\left(c_{\mathbf{r}_{A} \eta}^{s \dagger} c_{B_{l} \eta}^{z^{\prime}}+c_{\mathbf{r}_{A} \dagger}^{z^{\prime} \dagger} c_{B_{l} \eta}^{s}\right)\right. \\
& +X_{j}^{\prime l}\left(c_{\mathbf{r}_{A} x^{\prime} \dagger}^{x^{\prime}} c_{B_{l} \eta}^{z^{\prime}}-c_{\mathbf{r}_{A} \eta}^{z^{\prime} \dagger} c_{B_{l} \eta}^{x^{\prime}}\right) \\
& \left.+Y_{j}^{\prime l}\left(c_{\mathbf{r}_{A} \eta}^{y^{\prime} \dagger} c_{B_{l} \eta}^{z^{\prime}}-c_{\mathbf{r}_{A} \eta}^{z^{\prime} \dagger} c_{B_{l} \eta}^{y^{\prime}}\right)\right]+ \text { H.c., }
\end{aligned}
$$

where $c_{r \uparrow(\downarrow)}^{x^{\prime}}, c_{r \uparrow(\downarrow)}^{y^{\prime}}$, and $c_{r \uparrow(\downarrow)}^{z^{\prime}}$ represent the annihilation operators for $p_{x^{\prime}}, p_{y^{\prime}}, p_{z^{\prime}}$ orbital states with eigenspinor $\chi_{\uparrow(\downarrow)}$ along $\hat{\mathbf{z}}^{\prime}$ at a carbon atom $r$. Here, $S_{j}^{l}$, $X_{j}^{\prime l}$, and $Y_{j}^{\prime l}$ represent the curvature-induced coupling strength of $p_{x^{\prime}}, p_{y^{\prime}}$, and $s$ orbitals with a nearestneighbor $p_{z^{\prime}}$ orbitals. Their expressions are written as $S_{j}^{l}=\omega_{j}^{l} \tilde{S}_{j}^{l}, \quad X_{j}^{\prime l}=\omega_{j}^{l} \tilde{X}_{j}^{l} \cos \left[\theta_{j}\left(\mathbf{r}_{A}\right)\right]-\omega_{j}^{l} \tilde{Y}_{j}^{l} \sin \left[\theta_{j}\left(\mathbf{r}_{A}\right)\right]$, and $Y_{j}^{l l}=\omega_{j}^{l} \tilde{X}_{j}^{l} \sin \left[\theta_{j}\left(\mathbf{r}_{A}\right)\right]+\omega_{j}^{l} \tilde{Y}_{j}^{l} \cos \left[\theta_{j}\left(\mathbf{r}_{A}\right)\right]$, with $\omega_{j}^{1} \approx$ $a /\left(2 \mathcal{R}_{j}\left(\mathbf{r}_{A}\right)\right) \sin \left[\theta_{j}\left(\mathbf{r}_{A}\right)-\alpha\right], \omega_{j}^{2} \approx a /\left(2 \mathcal{R}_{j}\left(\mathbf{r}_{A}\right)\right) \sin [\pi / 3-$ $\left.\theta_{j}\left(\mathbf{r}_{A}\right)+\alpha\right]$, and $\omega_{j}^{3} \approx a /\left(2 \mathcal{R}_{j}\left(\mathbf{r}_{A}\right)\right) \sin \left[\pi / 3+\theta_{j}\left(\mathbf{r}_{A}\right)-\alpha\right]$. Here, $\tilde{S}_{j}^{l}, \tilde{X}_{j}^{l}$, and $\tilde{Y}_{j}^{l}$ are, respectively, written as

$$
\begin{aligned}
\tilde{S}_{j}^{1} & =V_{s p}^{\sigma} \sin \left[\beta_{j}\left(\mathbf{r}_{A}\right)\right], \\
\tilde{S}_{j}^{2} & =V_{s p}^{\sigma} \cos \left[\frac{\pi}{6}+\beta_{j}\left(\mathbf{r}_{A}\right)\right], \\
\tilde{S}_{j}^{3} & =V_{s p}^{\sigma} \cos \left[\frac{\pi}{6}-\beta_{j}\left(\mathbf{r}_{A}\right)\right], \\
\tilde{X}_{j}^{1} & =-V_{p p}^{\pi}-V_{p p}^{\pi} \cos ^{2}\left[\beta_{j}\left(\mathbf{r}_{A}\right)\right]-V_{p p}^{\sigma} \sin ^{2}\left[\beta_{j}\left(\mathbf{r}_{A}\right)\right], \\
\tilde{X}_{j}^{2} & =-V_{p p}^{\pi}-V_{p p}^{\pi} \cos ^{2}\left[\frac{\pi}{3}-\beta_{j}\left(\mathbf{r}_{A}\right)\right]-V_{p p}^{\sigma} \sin ^{2}\left[\frac{\pi}{3}-\beta_{j}\left(\mathbf{r}_{A}\right)\right], \\
\tilde{X}_{j}^{3} & =V_{p p}^{\pi}+V_{p p}^{\pi} \cos ^{2}\left[\frac{\pi}{6}-\beta_{j}\left(\mathbf{r}_{A}\right)\right]+V_{p p}^{\sigma} \sin ^{2}\left[\frac{\pi}{6}-\beta_{j}\left(\mathbf{r}_{A}\right)\right], \\
\tilde{Y}_{j}^{1} & =\frac{1}{2}\left(V_{p p}^{\pi}-V_{p p}^{\sigma}\right) \sin \left[2 \beta_{j}\left(\mathbf{r}_{A}\right)\right], \\
\tilde{Y}_{j}^{2} & =\frac{1}{2}\left(V_{p p}^{\pi}-V_{p p}^{\sigma}\right) \sin \left[2 \beta_{j}\left(\mathbf{r}_{A}\right)-\frac{2 \pi}{3}\right], \\
\tilde{Y}_{j}^{3} & =\frac{1}{2}\left(V_{p p}^{\pi}-V_{p p}^{\sigma}\right) \sin \left[2 \beta_{j}\left(\mathbf{r}_{A}\right)-\frac{\pi}{3}\right],
\end{aligned}
$$

where $\beta_{j}\left(\mathbf{r}_{A}\right)=\theta_{j}\left(\mathbf{r}_{A}\right)-\alpha$. Note that the local curvature generates interband transition between $\pi$-band and $\sigma$ band consisting of $s, p_{x}^{\prime}$, and $p_{y}^{\prime}$ orbitals $\frac{10 \mid 26 / 39}{}$ 
Secondly, $H_{\text {so }}$ is given by $H_{\text {so }}=\lambda_{\text {so }} \sum_{r} \mathbf{L}_{r} \cdot \mathbf{S}_{r}, \frac{10 / 40}{10}$ where $\mathbf{L}_{r}\left(\mathbf{S}_{r}\right)$ is atomic-orbital (spin) angular momentum of an electron at $r$, and can be expressed by $\underline{10126}$

$$
\begin{aligned}
H_{\mathrm{so}}= & \frac{\lambda_{\mathrm{so}}}{2} \sum_{r=\mathbf{r}_{A / B}}\left(c_{r \downarrow}^{z^{\prime} \dagger} c_{r \uparrow}^{x^{\prime}}-c_{r \uparrow}^{z^{\prime} \dagger} c_{r \downarrow}^{x^{\prime}}+i c_{r \uparrow}^{z^{\prime} \dagger} c_{r \downarrow}^{y^{\prime}}+i c_{r \downarrow}^{z^{\prime} \dagger} c_{r \uparrow}^{y^{\prime}}\right. \\
& \left.+i c_{r \uparrow}^{y^{\prime} \dagger} c_{r \uparrow}^{x^{\prime}}-i c_{r \downarrow}^{y^{\prime} \dagger} c_{r \downarrow}^{x^{\prime}}\right)+ \text { H.c.. }
\end{aligned}
$$

Note that on-site coupling between $p_{z^{\prime}}$ and $p_{x^{\prime}\left(y^{\prime}\right)}$ orbitals generates $\pi-\sigma$ interband transition that, combined with the curvature-induced interband transition, contributes to the $\mathcal{H}_{\text {soc }}(\mathbf{r})$.

In CNTs, however, the coupling between $p_{z^{\prime}}$ orbital and $p$ orbital along the CNT axis has no contribution to the effective SOC. For instance, if the Eq. (7) is expressed with eigenspinor along the CNT axis parallel to $\hat{\mathbf{y}}^{\prime}\left(\hat{\mathbf{x}}^{\prime}\right)$-direction instead of $\chi_{\uparrow(\downarrow)}$, the coupling between $p_{z^{\prime}}$ and $p_{y^{\prime}\left(x^{\prime}\right)}$ orbitals acquires a phase term $e^{ \pm i \phi}$ with the azimuthal angle $\phi$ along the circumference $e^{1026}$, and, as a result, its contribution averages out to zero after integration over the circumference $\frac{10}{10}$ Hence, the trivial extension from the curvature-induced SOC of the CNT to that of the SG is inevitably incomplete because of their topological difference in the geometrical structure.

Second-order process resulting from two consecutive $\pi-\sigma$ interband transitions gives rise to the effective SOC Hamiltonian for the unperturbed $\pi$-band states of Eq. (1) ${ }^{41}$ For the $\sigma$-band calculation, we use the SlaterKoster parametrization ${ }^{42}$ of $s, p_{x}$, and $p_{y}$ orbitals. During the second-order process, pseudospin-conserving and pseudospin-flipping processes occur; the former and the latter generate, respectively, the diagonal and the offdiagonal SOC in $\mathcal{H}_{\text {soc }}(\mathbf{r})$ [Eq. [2]] [26 The resulting effective SOC, then, is expressed in local coordinate axes of $\hat{\mathbf{x}}^{\prime}(\mathbf{r}), \hat{\mathbf{y}}^{\prime}(\mathbf{r}), \hat{\mathbf{z}}^{\prime}(\mathbf{r})$. However, for the smooth corrugation where $h_{i} h_{j} \ll 1(i, j=x, y), \hat{\mathbf{x}}^{\prime}(\mathbf{r}), \hat{\mathbf{y}}^{\prime}(\mathbf{r}), \hat{\mathbf{z}}^{\prime}(\mathbf{r})$ in the resulting effective SOC may be replaced by $\hat{\mathbf{x}}, \hat{\mathbf{y}}, \hat{\mathbf{z}}$ to obtain $\mathcal{H}_{\mathrm{soc}}(\mathbf{r})$ in Eq. (2) since the difference between the two sets of axes [for instance, $\hat{\mathbf{z}}^{\prime}(\mathbf{r}) \cdot \hat{\mathbf{z}}=\left(1+|\nabla h(\mathbf{r})|^{2}\right)^{-\frac{1}{2}} \approx 1$ ] generates higher order terms in $a / \mathcal{R}_{1(2)}(\mathbf{r})$.

Lastly, we remark $d$ orbitals effects on the curvatureinduced SOC, which were not considered in our calculation. For the spin-orbit induced gap, the $d$ orbital effects are recently reported to be important since the hybridization between $d$ orbitals and $p$ orbitals generate the gap, which is linear in the atomic SOC strength $\lambda_{d}$ of $d$ orbitals. ${ }^{37 / 38}$ This linear contribution can be dominant over the $p$ orbital contribution, which is quadratic in $\lambda_{\text {so }}$. In contrast, the contribution of the $d$ orbitals to the external field-induced Rashba SOC is smaller than that of the $p$ orbitals ${ }^{38}$ The coupling between $p$ and $d$ orbitals can also lead to the curvature-induced SOC in the first order of $a / \mathcal{R}_{1(2)}(\mathbf{r})$. Due to the symmetrical reason, however, its form is expected to be same as the form of $\mathcal{H}_{\text {soc }}(\mathbf{r})$ [Eq. 22)] except parameters in $\zeta$ and $\zeta^{\prime}$. The curvatureinduced SOC owing to the $d$ orbitals can be generated as follows. The $\pi$-band consisting of the $p_{z}$ orbital and the nearest-neighbor $d_{x z}$ and $d_{y z}$ orbitals can couple with the $\sigma$ bands through on-site coupling between $d$ orbitals in $\pi$-band and $\sigma$-band by the atomic SOC. Then, electrons in $\sigma$-band can delocalize to the nearest-neighbor $p$ and $d$ orbitals within $\sigma$ bands. Finally, the $p$ and $d$ orbitals in $\sigma$-bands can couple with the nearest-neighbor $p_{z}$ orbitals due to the curvature, yielding the curvature-induced SOC that is proportional to $a / \mathcal{R}_{1(2)}(\mathbf{r}), \lambda_{d}$, and $\left(\epsilon_{d}-\epsilon_{p}\right)^{-2}$. Based on the estimation of the Rashba SOC strength and comparison with the $p$ orbital contribution given in Ref. ${ }^{[38}$, we can compare roughly the $d$ orbital contribution of the curvature-induced SOC with the $\mathcal{H}_{\text {soc }}(\mathbf{r})$ [Eq. 2 . The $\lambda_{d}$ is smaller than $\lambda_{\mathrm{so}}$, and further, $\left(\epsilon_{d}-\epsilon_{p}\right)^{-2}$ multiplied by hopping parameters associated with $d$ and $p$ orbitals is a small quantity compared with parameters in same dimension in $\zeta$ and $\zeta^{\prime}$. Hence, we believe that the contribution of $d$ orbitals to the curvature-induced SOC is smaller than that of $p$ orbitals. In order to get more reliable quantitative estimates, systematic study considering coupling between $p, s$ orbitals and $d$ orbitals is required ${ }^{38}$

\section{SPIN RELAXATION}

In this section, we investigate effects of the geometric curvature on SR in chemically clean SGs with nanoscale corrugations. Motivated by recent experiments ${ }^{14}$ about the corrugation roughness of SGs, we study SR dependence on the fractal dimension of the corrugation roughness within the assumption that both SOC and momentum scattering ${ }^{30}$ arise mainly from the corrugation. Since the substrate-induced SOC effect on SR was addressed in a previous theory, ${ }^{[9]}$ we focus on the curvatureinduced SOC here.

\section{A. Kinetic equation of the real spin density opeator}

In order to calculate SRT, we use the kinetic equation of the real spin density operator $\rho .22 \mid 43$ Besides the $\mathcal{H}_{\text {soc }}(\mathbf{r})$, we include the strain-induced vector potential $\mathcal{H}_{\mathrm{v}}(\mathbf{r})$ that occurs in corrugated SGs 44 46 $\mathrm{Al}-$ though $\mathcal{H}_{\mathrm{v}}(\mathbf{r})$ is independent of the spin, it is still relevant for the SRT since it affects the momentum scattering rate. Then the local potential Hamiltonian $\mathcal{H}_{\mathrm{p}}(\mathbf{r})$ is written as $\mathcal{H}_{\mathrm{p}}(\mathbf{r}) \equiv \mathcal{H}_{\text {soc }}(\mathbf{r})+\mathcal{H}_{\mathrm{v}}(\mathbf{r})$ which is considered as weak perturbation in the kinetic equation. We assume $\left\langle\mathcal{H}_{\mathrm{p}}(\mathbf{r})\right\rangle \approx 0$ where $\langle\cdots\rangle$ denotes ensemble average. Since $\mathcal{H}_{\mathrm{p}}(\mathbf{r})$ is expected to change slowly over scales larger than the lattice spacing so that the hybridization between $\tau_{z}=1$ and $\tau_{z}=-1$ states can be ignored, we calculate SRT for the electron state of the $\mathcal{H}_{\text {Dirac }}$ [Eq. (1)] when $\tau_{z}=1$ and $\alpha=0$, where eigenenergy $\varepsilon_{\mathbf{k}}$ and eigenstate $\left|\psi_{\mathbf{k}}\right\rangle$ are, respectively, given by $\varepsilon_{\mathbf{k}}=\hbar v_{F} k$ with $k \equiv|\mathbf{k}|$, and $\left|\psi_{\mathbf{k}}\right\rangle=1 / \sqrt{2}\left(e^{-i \varphi_{\mathbf{k}}} 1\right)^{\mathrm{T}}|\mathbf{k}\rangle$ with polar angle 
$\varphi_{\mathbf{k}}$ of the wavevector $\mathbf{k} .^{[4]}$ Then a $2 \times 2$ real spin density operator $\rho_{\mathbf{k}}$ diagonal in momentum has a representation in eigenspinors chosen to be along $\hat{\mathbf{z}}$ direction. The kinetic equation of $\rho_{\mathbf{k}}$ is written as ${ }^{22 / 43}$

$$
\frac{\partial \rho_{\mathbf{k}}}{\partial t}+\frac{i}{\hbar}\left[V_{\mathbf{k k}}, \rho_{\mathbf{k}}\right]=\mathcal{C}_{\mathbf{k}}
$$

where $V_{\mathbf{k k}^{\prime}}=\left\langle\psi_{\mathbf{k}}\left|\mathcal{H}_{\mathrm{p}}(\mathbf{r})\right| \psi_{\mathbf{k}^{\prime}}\right\rangle$, and $\mathcal{C}_{\mathbf{k}}$ is the collision integral describing momentum scattering, which is given by $\mathcal{C}_{\mathbf{k}}=\pi / \hbar \sum_{\mathbf{k}^{\prime}}\left[2 V_{\mathbf{k k}^{\prime}} \rho_{\mathbf{k}^{\prime}} V_{\mathbf{k}^{\prime} \mathbf{k}}-\left\{\rho_{\mathbf{k}}, V_{\mathbf{k k}^{\prime}} V_{\mathbf{k}^{\prime} \mathbf{k}}\right\}\right] \delta\left(\varepsilon_{\mathbf{k}^{\prime}}-\varepsilon_{\mathbf{k}}\right)$. The commutator in Eq. (8) can be ignored because $\mathcal{H}_{\mathrm{p}}(\mathbf{r})$ has no regular contribution. Since the energy scale of $\mathcal{H}_{\mathrm{v}}(\mathbf{r})$ is typically larger than that of $\mathcal{H}_{\mathrm{soc}}(\mathbf{r})$, the SRT would be longer than a time scale in which the momentum distribution during momentum scattering becomes isotropic. ${ }^{43}$ Thus, the $\rho_{\mathbf{k}}$ can be represented as $\rho_{\mathbf{k}}=\bar{\rho}+\rho_{\mathbf{k}}^{\prime}$ with the anisotropic part $\rho_{\mathbf{k}}^{\prime}$ due to the $\mathcal{H}_{\text {soc }}(\mathbf{r})$, where the bar means averaging over $\varphi_{\mathbf{k}}$. Inserting the $\rho_{\mathbf{k}}=\bar{\rho}+\rho_{\mathbf{k}}^{\prime}$ into Eq. (8), we obtain the equation of $\bar{\rho}$ as,

$$
\frac{\partial \bar{\rho}}{\partial t}=\frac{\pi d}{\hbar} \oint d \varphi_{\mathbf{k}} \oint d \varphi_{\mathbf{k}^{\prime}}\left(V_{\mathbf{k k}^{\prime}}^{\prime} \bar{\rho} V_{\mathbf{k}^{\prime} \mathbf{k}}^{\prime}-\frac{1}{2}\left\{\bar{\rho},\left|V_{\mathbf{k k}^{\prime}}^{\prime}\right|^{2}\right\}\right),
$$

where $d=k /\left(\pi \hbar v_{F}\right)$ is the density of states. Note that collision term consisting of the product of $\mathcal{H}_{\mathrm{soc}}(\mathbf{r})$ and $\mathcal{H}_{\mathrm{v}}(\mathbf{r})$ disappears since there is no correlation between them. More specifically, since the vector potential is written as quadratic in $h_{i, j}, \frac{4445}{\left\langle h_{i}\right.}$ the collision terms proportional to $\left\langle h_{i} h_{j} \boldsymbol{\mu}(\mathbf{r})\right\rangle$ or $\left\langle h_{i} h_{j} \nu(\mathbf{r})\right\rangle$ vanish by the Wick theorem. ${ }^{30}$ Thus, the random gauge field does not contribute to the SR in chemically clean SGs $\underline{48}$

Since the real spin density $\mathbf{P}=\left(P_{x}, P_{y}, P_{z}\right)$ can be evaluated by $\mathbf{P}=\operatorname{Tr}(\bar{\rho} \mathbf{s})$, we can obtain the kinetic equation of $\mathbf{P}$ by tracing Eq. (9) multiplied by $\mathbf{s}$ over the real spin space, which is written as $\partial P_{i} / \partial t \approx-P_{i} / \tau_{i}^{s}(i=x, y, z)$ with $\tau_{i}^{s}$ being the SRT of spin along the $i$-direction. Here, $\tau_{x(y)}^{s}$ and $\tau_{z}^{s}$ are evaluated as

$$
\frac{1}{\tau_{x(y)}^{s}}=\frac{4 \pi^{3} d}{\hbar} \iint d \mathbf{r} d \mathbf{r}^{\prime}\left[\zeta^{\prime 2}\left\langle\mu_{y(x)}(\mathbf{r}) \mu_{y(x)}\left(\mathbf{r}^{\prime}\right)\right\rangle J+\zeta^{2}\left\langle\nu(\mathbf{r}) \nu\left(\mathbf{r}^{\prime}\right)\right\rangle J^{\prime}\right]
$$

and $1 / \tau_{z}^{s}=1 / \tau_{x}^{s}+1 / \tau_{y}^{s}$, where $J$ and $J^{\prime}$ are, respectively, defined as $J \equiv J_{0}^{2}(k R)+J_{1}^{2}(k R)$, and $J^{\prime} \equiv J_{0}^{2}(k R)$ with $J_{0(1)}(x)$ being the Bessel function of zeroth (first) order and $R=\left|\mathbf{r}-\mathbf{r}^{\prime}\right|$. If we assume $\left\langle\mu_{x}(\mathbf{r}) \mu_{x}\left(\mathbf{r}^{\prime}\right)\right\rangle \approx$ $\left\langle\mu_{y}(\mathbf{r}) \mu_{y}\left(\mathbf{r}^{\prime}\right)\right\rangle$ and $\left\langle\mu_{x}(\mathbf{r}) \mu_{y}\left(\mathbf{r}^{\prime}\right)\right\rangle \approx 0$ for the random corrugation, we obtain $\tau_{x}^{s} \approx \tau_{y}^{s}$ and $\tau_{x(y)}^{s} \approx 2 \tau_{z}^{s}$, which means that spins out of plane relax twice as fast as spins in plane.$^{20}$ Note that since the carrier density $n$ is given by $n=k^{2} / \pi$, the SR vanishes at the Dirac points where $n=0 \underline{22}$

\section{B. Numerical results}

Figure 4 shows SRTs, $\left\langle\tau_{z}^{s}\right\rangle$ that are calculated numerically in corrugated SG surfaces for $\alpha=0$ and are ensemble-averaged for ten surfaces. Those surfaces are approximated as square lattices, which can be justified since the $\mathcal{H}_{\text {soc }}(\mathbf{r})$ contains the curvature effects taking into account the honeycomb lattice. The surface with fluctuating $h(\mathbf{r})$ is constructed based on the heightheight correlation function $g(R)=\left\langle\left(h(\mathbf{r})-h\left(\mathbf{r}^{\prime}\right)\right)^{2}\right\rangle=$ $2 \gamma^{2}\left(1-e^{-(R / \xi)^{2 H}}\right)$, where $H$ characterizes the fractal dimension, $14[30 \mid 49] \xi$ represents the correlation length, and $\gamma=\sqrt{\left\langle h^{2}(\mathbf{r})\right\rangle}$. As the fractal dimension $H$ increases, the corrugated surface becomes smooth.

A recent experiment ${ }^{12}$ in suspended SGs shows that the height of corrugations grows approximately linearly with increasing length, which corresponds to a corrugated surface for $2 H=2$. For exfoliated SGs on the substrate, on the other hand, the fractal dimension with
$2 H \approx 1$ was observed 14 There is a controversy over the origin of observed corrugation roughness ${ }^{16}$ Here, we in-

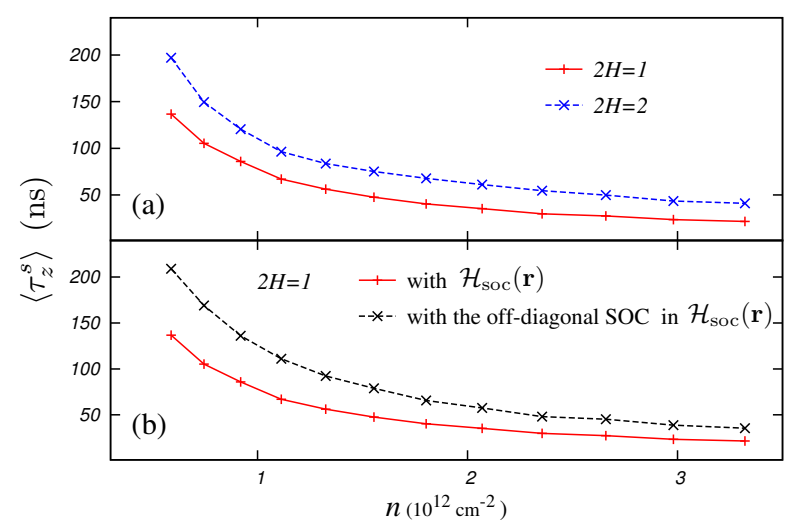

FIG. 4. (Color online) Spin relaxation time $\left\langle\tau_{z}^{s}\right\rangle$ as a function of the carrier density $n=k^{2} / \pi$ and the fractal dimension $H$. The $\left\langle\tau_{z}^{s}\right\rangle$ are ensemble-averaged quantities over ten surfaces for $\alpha=0$. The corrugated square lattice with the height $h(\mathbf{r})$, the lattice constant of $0.2 \mathrm{~nm}$, and the side length of $200 \mathrm{~nm}$, are constructed numerically based on the random midpoint displacement method $\sqrt{50}$ with height-height correlation function $g(R)=2 \gamma^{2}\left(1-e^{-(R / \xi)^{2 H}}\right)$. Here, we set $\gamma=0.3 \mathrm{~nm}$ and $\xi=20 \mathrm{~nm}$ from recent experiments, $\frac{14[15}{15}$ and interpolate discrete $h(\mathbf{r})$ in order to mimic the continuous SG surface. (a) $\left\langle\tau_{z}^{s}\right\rangle$ as a function of $n$ for $2 H=1$ (red solid line) and $2 H=2$ (blue dashed line). (b) $\left\langle\tau_{z}^{s}\right\rangle$ as a function of $n$ for $2 H=1$ considering $\mathcal{H}_{\text {soc }}(\mathbf{r})$ (red solid line) and only the off-diagonal SOC in $\mathcal{H}_{\text {soc }}(\mathbf{r})$ (black dashed line), respectively. 
vestigate $\mathrm{SR}$ in corrugated SGs for $2 H=1$ and $2 H=2$ representatively. As shown in Fig. 4(a), the $\left\langle\tau_{z}^{s}\right\rangle$ becomes shorter with decreasing $2 H$, which can be understood in terms of a growing irregularity of $\mathcal{H}_{\mathrm{soc}}(\mathbf{r})$ [Eq. 2] induced by rougher surface corrugation. Also, we check that the $\left\langle\tau_{x(y)}^{s}\right\rangle$ is approximately twice as large as the $\left\langle\tau_{z}^{s}\right\rangle$ (not shown here).

Next, in order to investigate the effect of the diagonal SOC in $\mathcal{H}_{\text {soc }}(\mathbf{r})$, we compare two SRTs, one calculated considering the entire $\mathcal{H}_{\text {soc }}(\mathbf{r})$ and the other considering only the off-diagonal SOC term in $\mathcal{H}_{\text {soc }}(\mathbf{r})$, respectively, in SGs with $2 H=1$. As shown in Fig. 4 (b), the latter SRT is noticeably larger than the former true SRT. Hence, it is necessary to consider both SOC terms for the precise analysis of SR depending on the corrugation roughness.

The calculated SRT as shown in Fig. 4 is at least of the order $\sim 10 \mathrm{~ns}$, and becomes shorter monotonically as the carrier density $n$ increases, which is primarily because the density of states $d$ increases linearly with $n^{1 / 2}$ [Eq. (10)]. We check that the integrand of Eq. (10) has little dependence relatively upon $k$. This implies that the effective spatial range of the random SOC affecting the SR substantially does not depend significantly on the variation of $k$ over the scale $\sim 10 \mathrm{~nm}^{-1}$. Our quantitative and qualitative results are in agreement with a recent theory ${ }^{[22}$ As mentioned in the Ref. ${ }^{22}$, however, those results cannot explain recent spin transport experiments. 4

One possibility for that discrepancy is that the effect of charged impurity in the substrate causing momentum scattering $\sqrt{51}$ is not considered in our calculation. The momentum scattering combined with the curvatureinduced Rashba-type SOC was already investigated theoretically, $\sqrt{20}$ but it cannot explain the measured SRT, either. However, the inclusion of the additional diagonal SOC in $\mathcal{H}_{\text {soc }}(\mathbf{r})$ in addition to the off-diagonal SOC into the EY mechanism could give a possibility to calculate the SRT more precisely. In the EY mechanism, ${ }^{[7}$ spin flip scattering occurs at the very moment when the momentum scattering takes place since the electron wave functions normally have an admixture of the opposite-spin states due to the SOC.

\section{CONCLUSION}

We have demonstrated that the combined action of the curvature and the atomic SOC of carbon gives rise to two types of the effective SOC in SGs with corrugation or periodic ripples. One of them couples with the sublattice pseudospin, which corresponds to the Rashba-type SOC in a corrugated SG reported in previous theories, 10 20 22 . The other SOC, on the other hand, does not couple with the pseudospin, and was not recognized in previous literature. The additional curvature-induced SOC depends on the principal curvature direction, which is similar to the curvature-induced SOC of CNTs whose diagonal term in pseudospin representation depends on the chiral angle of CNTs. However, the curvature-induced SOC in SGs can not be obtained from the trivial extension of the curvature-induced SOC in CNTs due to their distinct topological structure between the SG surface and the CNT cylinder.

We have also investigated SR in chemically clean SGs with nanoscale corrugation, and found that the diagonal SOC makes the same order of contribution to SRT as the off-diagonal SOC. The SRT becomes longer as the fractal dimension of the corrugation roughness increases since the irregularity of the SOC decreases in smoother SGs. The calculated SRT in chemically clean SGs, however, can not explain recent experimental results of SR in current exfoliated SG samples.

A natural direction for future research would be to calculate SRT in the presence of charged impurities that cause momentum scattering, ${ }^{51}$ considering both the diagonal and the off-diagonal SOC. In addition to the SR in SGs, we expect that the curvature-induced SOC $\left[\mathcal{H}_{\text {soc }}(\mathbf{r})\right]$ may be relevant for the analysis of other spin-related phenomena in SGs.

\section{ACKNOWLEDGMENTS}

We thank D. Gang, T. Takimoto, S. Kettemann, K. Kim, B. H. Kim, J. Han, I. Kim, S. Kim, K. Hashimoto, F. Ziltener, Y.-W. Son, S.-M. Choi, and S. Lee for useful discussions. This work was financially supported by the NRF (2010-0014109, 2011-0030790) and BK21.
* jsjeong@kias.re.kr

1 A. H. Castro Neto, F. Guinea, N. M. R. Peres, K. S. Novoselov, and A. K. Geim, Rev. Mod. Phys. 81, 109 (2009); A. K. Geim, Science 324, 1530 (2009).

2 Y.-W. Son, M. L. Cohen, and S. G. Louie, Nature (London) 444, 347 (2006). W. Y. Kim and K. S. Kim, Nat. Nanotechnol. 3, 408 (2008).

3 B. Trauzettel, D. V. Bulaev, D. Loss, and G. Burkard, Nat. Phys. 3, 192 (2007); P. Recher and B. Trauzettel, Nanotechnology 21, 302001 (2010).

${ }^{4}$ N. Tombros, C. Jozsa, M. Popinciuc, H. T. Jonkman, and
B. J. van Wees, Nature (London) 448, 571 (2007); N. Tombros, S. Tanabe, A. Veligura, C. Jozsa, M. Popinciuc, H. T. Jonkman, and B. J. van Wees, Phys. Rev. Lett. 101, 046601 (2008); C. Józsa, T. Maassen, M. Popinciuc, P. J. Zomer, A. Veligura, H. T. Jonkman, and B. J. van Wees, Phys. Rev. B 80, 241403(R) (2009); W. Han, and R. K. Kawakami, Phys. Rev. Lett. 107, 047207 (2011); S. Jo, D.-K. Ki, D. Jeong, H.-J. Lee, and S. Kettemann, Phys. Rev. B 84, 075453 (2011); W. Han, K. M. McCreary, K. Pi, W. H. Wang, Y. Li, H. Wen, J. R. Chen, and R. K. Kawakami, J. Magn. Magn. Mater. 324, 369 (2012). 
${ }^{5}$ F. J. Jedema, H. B. Heersche, A. T. Filip, J. J. A. Baselmans, and B. J. van Wees, Nature (London) 416, 713 (2002).

${ }^{6}$ F. J. Jedema, M. S. Nijboer, A. T. Filip, and B. J. van Wees, Phys. Rev. B 67, 085319 (2003).

7 I. Žutić, J. Fabian, S. Das Sarma, Rev. Mod. Phys. 76, 323 (2004).

8 A. H. Castro Neto and F. Guinea, Phys. Rev. Lett. 103, 026804 (2009).

9 C. Ertler, S. Konschuh, M. Gmitra, and J. Fabian, Phys. Rev. B 80, 041405 (R) (2009).

10 D. Huertas-Hernando, F. Guinea, and A. Brataas, Phys. Rev. B 74, 155426 (2006).

11 P. Zhang and M. W. Wu, Phys. Rev. B 84, 045304 (2011); H. Ochoa, A. H. Castro Neto, and F. Guinea, arXiv:1107.3386v2 (2011); P. Zhang, and M. W. Wu, arXiv:1108.0283 2 (2011).

12 J. C. Meyer, A. K. Geim, M. I. Katsnelson, K. S. Novoselov, T. J. Booth, and S. Roth, Nature (London), 446, 60 (2007).

13 E. Stolyarova, K. T. Rim, S. Ruy, J. Maultzsch, P. Kim, L. E. Brus, T. F. Heinz, M. S. Hybertsen, and G. W. Flynn, Proc. Natl. Acad. Sci. U.S.A. 104, 9209 (2007).

14 M. Ishigami, J. H. Chen, W. G. Cullen, M. S. Fuhrer, and E. D. Williams, Nano Lett. 7, 1643 (2007).

15 V. Geringer, M. Liebmann, T. Echtermeyer, S. Runte, M. Schmidt, R. Rückamp, M. C. Lemme, and M. Morgenstern, Phys. Rev. Lett. 102, 076102 (2009).

16 W. G. Cullen, M. Yamamoto, K. M. Burson, J. H. Chen, C. Jang, L. Li, M. S. Fuhrer, and E. D. Williams, Phys. Rev. Lett. 105, 215504 (2010).

17 K. R. Knox, A. Locatelli, M. B. Yilmaz, D. Cvetko, T. O. Menteş, M. Á. Niño, P. Kim, A. Morgante, and R. M. Osgood Jr., Phys. Rev. B 84, 115401 (2011).

18 W. Bao, F. Miao, Z. Chen, H. Zhang, W. Jang, C. Dames, and C. N. Lau, Nat. Nanotechnol. 4, 562 (2009).

19 J. S. Choi, J.-S. Kim, I.-S. Byun, D. H. Lee, M. J. Lee, B. H. Park, C. Lee, D. Yoon, H. Cheong, K. H. Lee, Y.W. Son, J. Y. Park, and M. Salmeron, Science, 333, 607 (2011).

20 D. Huertas-Hernando, F. Guinea, and A. Brataas, Phys. Rev. Lett. 103, 146801 (2009).

21 Y. Zhou and M. W. Wu, Phys. Rev. B 82, 085304 (2010).

${ }^{22}$ V. K. Dugaev, E. Ya. Sherman, and J. Barnaś, Phys. Rev. B 83, 085306 (2011).

${ }^{23}$ F. Kuemmeth, S. Ilani, D. C. Ralph, and P. L. McEuen, Nature (London) 452, 448 (2008).

${ }^{24}$ T. Ando, J. Phys. Soc. Jpn. 69, 1757 (2000).

${ }^{25}$ W. Izumida, K. Sato, and R. Saito, J. Phys. Soc. Jpn. 78, 074707 (2009).

26 J.-S. Jeong and H.-W. Lee, Phys. Rev. B 80, 075409 (2009).

27 T. S. Jespersen, K. Grove-Rasmussen, J. Paaske, K. Muraki, T. Fujisawa, J. Nygård, and K. Flensberg, Nat. Phys. 7, 348 (2011).

28 A. Fasolino, J. H. Los, and M. I. Katsnelson, Nature Mater. 6, 858 (2007).

${ }^{29}$ It was reported recently that the corrugation can be suppressed if the SG is exfoliated onto mica surfaces [C. H.
Lui, L. Lui, K. F. Mak, G. W. Flynn, and T. F. Heinz, Nature (London), 462, 339 (2009)].

30 M. I. Katsnelson and A. K. Geim, Phil. Trans. R. Soc. A 366, 195 (2008).

31 G. W. Semenoff, Phys. Rev. Lett. 53, 2449 (1984); D. P. DiVincenzo and E. J. Mele, Phys. Rev. B 29, 1685 (1984).

32 J. Serrano, M. Cardona, and T. Ruf, Solid State Commun. 113, 411 (2000).

33 C. L. Kane, and E. J. Mele, Phys. Rev. Lett. 95, 226801 (2005).

34 D. Tománek and M. A. Schluter, Phys. Rev. Lett. 67, 2331 (1991).

35 D. J. Struik, Lectures on Classical Differential Geometry, 2nd ed. (Dover, New York, 1988).

${ }^{36}$ Leonard I. Schiff, Quantum Mechanics (McGraw-Hill, New York, 1968).

37 M. Gmitra, S. Konschuh, C. Ertler, C. Ambrosch-Draxl, and J. Fabian, Phys. Rev. B 80, 235431 (2009).

38 S. Konschuh, M. Gmitra, and J. Fabian, Phys. Rev. B 82, 245412 (2010).

39 E.-A. Kim and A. H. Castro Neto, Europhys. Lett. 84, 57007 (2008).

${ }^{40}$ H. Min, J. E. Hill, N. A. Sinitsyn, B. R. Sahu, L. Kleinman, and A. H. MacDonald, Phys. Rev. B 74, 165310 (2006); Y. Yao, F. Ye, X.-L. Qi, S.-C. Zhang, and Z. Fang, ibid 75, 041401(R) (2007).

41 The comprehensive explanation of the degenerate secondorder perturbation theory is described well in Ref! ${ }^{10[26}$.

42 J. C. Slater and G. F. Koster, Phys. Rev. 94, 1498 (1954).

43 Optical Orientation, edited by F. Mayer and B. Zakharchenya (North-Holland, Amsterdam, 1984); N. S. Averkiev, L. E. Golub, and M. Willander, J. Phys.:Condens. Matter 14, R271 (2002); S. A. Tarasenko, JETP Letters 84, 199 (2006).

44 T. Ando, J. Phys. Soc. Jpn. 75, 124701 (2006),

45 S. V. Morozov, K S. Novoselov, M. I. Katsnelson, F. Schedin, L. A. Ponomarenko, D. Jiang, and A. K. Geim, Phys. Rev. Lett. 97, 016801 (2006).

46 F. Guinea, B. Horovitz, and P. Le Doussal, Phys. Rev. B 77, 205421 (2008).

47 We evaluate SRT for hole states of Eq. 11 as well, and obtain electron-hole symmetric SRT.

48 A strain-induced vector potential arising from in-plane atomic displacement $\mathrm{t}^{44 \mid 45}$ is not correlated with the $\mathcal{H}_{\text {soc }}(\mathbf{r})$ that is dependent on the curvature only. In addition, the scalar potential induced by the curvature effects ${ }^{39}$ is quadratic in local mean curvature.

49 B. B. Mandelbrodt, The Fractal Geometry of Nature (Freeman, New York, 1982); G. Palasantzas, Phys. Rev. B 48, 14472 (1993).

50 A. Fournier, D. Fussel, and L. Carpenter, Communications of the ACM, 25, 371 (1982).

51 T. Ando, J. Phys. Soc. Jpn. 75, 074716 (2006); K. Nomura and A. H. MacDonald, Phys. Rev. Lett. 96, 256602 (2006); K. Nomura and A. H. MacDonald, ibid 98, 076602 (2007); E. H. Hwang, S. Adam, and S. Das Sarma, ibid 98, 186806 (2007); S. Adam, E. H Hwang, V. Galitski, and S. Das Sarma, Proc. Natl. Acad. Sci. U. S. A. 104, 18392 (2007). 